\title{
Ultrasound-guided percutaneous irrigation of rotator cuff calcific tendinosis. What radiologist should know
}

\author{
Vito Chianca ${ }^{1, E, F}$, Francesco Di Pietto ${ }^{2, B}$, Domenico Albano ${ }^{3,4, F}$, Antonio Corvino ${ }^{5, F}$, Filippo Del Grande ${ }^{1, E, F}$ \\ 'Clinica di Radiologia EOC IIMSI, Lugano, Switzerland \\ 2Department of Radiology, Pineta Grande Hospital, Castel Volturno, CE, Italy \\ ${ }^{3}$ RCCS Istituto Ortopedico Galeazzi, Milano, Italy \\ ${ }^{4}$ Sezione di Scienze Radiologiche, Dipartimento di Biomedicina, Neuroscienze e Diagnostica Avanzata, Università degli Studi di Palermo, Italy \\ 5Motor Science and Wellness Department, University of Naples "Parthenope", Naples, Italy
}

\section{Abstract}

\begin{abstract}
Rotator cuff calcific tendinopathy is a common condition caused by the presence of calcification into the rotator cuff or in the subacromial-subdeltoid bursa. The pathogenetic mechanism of this pathology is still debated. Calcific tendinitis frequently affects the rotator cuff and may cause shoulder pain and reduction of range of motion. It can be diagnosed with conventional radiography, ultrasound, or magnetic resonance imaging. The first therapeutic option includes conservative management based on rest, physical therapy, and oral non-steroid anti-inflammatory administration. Extracorporeal shock wave therapy is a noninvasive technique that can be useful for the fragmentation of calcific deposits. Imaging-guided percutaneous irrigation is currently considered the gold standard technique for the treatment of calcific tendinitis due to its minimal invasiveness and its success rate of about $80 \%$.
\end{abstract}

Key words: calcific tendinopathy, US, MRI, percutaneous treatments.

\section{Introduction}

Rotator cuff calcific tendinitis (RCCT) is a very common pathology, which presents in up to $7.5 \%$ of asymptomatic adults and up to $17 \%$ of patients with shoulder pain, caused by the deposition of calcium in the rotator cuff (RC) tendons [1]. This clinical condition occurs frequently in women, in about $60-70 \%$ of cases, especially between their $4^{\text {th }}$ and $5^{\text {th }}$ decades of life, and in $20 \%$ of cases the deposits are bilateral [2]. In 15-20\% of patients, small deposits are usually asymptomatic, but symptoms, when present, may vary from sub-acute pain that worsens during the night to a severe and disabling condition associated with restriction of range of movements (ROM) resistant to anti-inflammatory drugs [3].
All tendons of the body may be affected by CT, but RC is the most involved site, and in particular [4]:

- the supraspinatus, about $1 \mathrm{~cm}$ from its tendinous insertion on the grater humeral tuberosity, the so-called "critical zone area" (80\%);

- the lower side of the infraspinatus (15\%);

- the pre-insertional portion of sub-scapularis tendon (5\%).

This clinical condition tends to resolve, often spontaneously. However, no consensus exists regarding treatment options. Patients with low-grade symptoms may be treated conservatively with rest, physical therapy, and oral non-steroid anti-inflammatory (NSAIDs) drugs while in patients with severe symptoms surgery can be replaced by new, less invasive techniques such as extracorporeal shock wave therapy (ESWT) and ultrasound-

Correspondence address:

Vito Chianca, MD, Clinica di Radiologia EOC IIMSI, Lugano, Switzerland, e-mail: vitochianca@gmail.com

Authors' contribution:

A Study design · B Data collection · C Statistical analysis · D Data interpretation · E Manuscript preparation · F Literature search · G Funds collection 
guided percutaneous irrigation of calcific tendinopathy (US-PICT) [5].

\section{Aetiology and pathogenesis}

The aetiology of CT remains largely unclear with multiple theories proposed. The process is probably related to a metaplastic transformation of tenocytes into chondrocytes that induce the deposition of calcific crystals inside the tendons [6]. Some authors correlate CT with a reduction of intratendinous oxygen afflux that may promote fibrosis and necrosis with subsequent fibre degeneration followed by calcific deposition [7]. Bishop and Bosworth individually proposed a theory based on the concept that repetitive tendons microtrauma leads to fibre degeneration followed by calcification $[2,8]$. Rui et al. postulated a relatively new theory based on an erroneous differentiation of tendon-derived stem cells (TDSCs) into bone cells, which leads to chondral metaplasia [9]. Recent studies focus their attention on the potential role of chondroosteogenic BMP-2, BMP-4, and BMP-7 in the metaplasia of tendon cells leading to calcification [10].

According to Uhthoff et al., RCCT can be divided into 4 stages [11]:

- first stage: precalcific stage, in which tendon metaplastic transformation in fibrocartilaginous tissue acts as a substrate for calcific deposits;

- second stage: calcific formative stage, in which there is formation of single or multiple crystal deposition inside the tendons;

- third stage: calcific resorptive stage, in which an increase in vascularity and oedematous infarction is seen with subsequent macrophages phagocytosis of deposits; the resorptive stage is usually associated with acute symp-

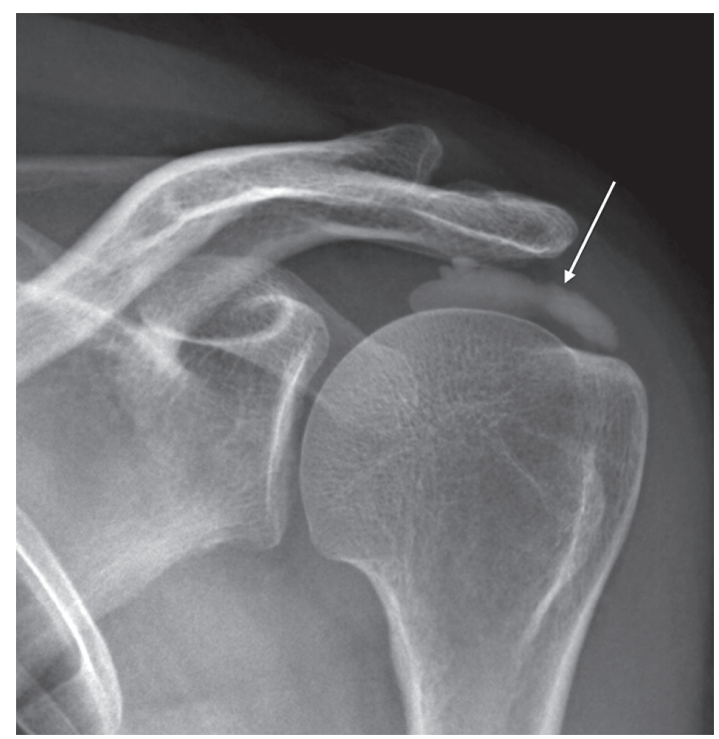

Figure 1. Conventional radiographic evaluation of left shoulder showing cloudy and dense calcification (arrow) of the supraspinatus/infraspinatus projective sites toms due to the oedema, which causes increased intratendinous pressure and, in some cases, the extravasation of crystals in the sub acromion subdeltoid bursa (SASD);

- fourth stage: post-calcific stage: in which self-healing tendon repair by fibroblast requires several months. In that phase patients may present severe pain with reduction of ROM.

\section{Imaging findings}

\section{Conventional radiography}

Conventional radiography (CR) is usually the first imaging modality for the investigation of shoulder pain. When RCCT is suspected, CR may detect the presence of calcifications in the projective site of the rotator cuff or in the subacromial space that confirms the diagnosis (Figure 1).

The standard shoulder CR exam includes true anteroposterior view in internal, external, and neutral rotation of the arm and outlet view; although, an additional axillary lateral view can be helpful to detect calcification of subscapularis tendon [12].

Several classifications have been proposed based on the morphology, size, and radiographic aspect (Table 1), but all of them lack sufficient reproducibility [13].

\section{Ultrasound}

Ultrasound (US) is an established modality for the investigation of soft tissue pathologies [14]. On US, calcific deposits usually appear hyperechoic with or without acoustic posterior shadowing, due to the variable amount of calcific content [15]. Several classifications have been proposed in the literature but the 2 most important are Bianchi-Martinoli and Sconfienza:

Bianchi and Martinoli [16] described different calcification morphologies, based on the percentage of calcium: - type I calcification: hyperechoic foci with a well-defined acoustic shadowing;

- type II calcification: hyperechoic foci with a mild acoustic shadow due to the lower amount of calcium deposit;

Table 1. Classification of calcific deposits

Mole et al. classification

\begin{tabular}{|c|l|}
\hline Type a & Sharply, homogenous and dense \\
\hline Type b & Sharply, defined, dense with fragments \\
\hline Type c & Heterogeneous with soft contours \\
\hline Type d & $\begin{array}{l}\text { Dystrophic calcifications at the insertion of the rotator } \\
\text { cuff tendons }\end{array}$ \\
\hline
\end{tabular}

Gartner and Heyer classification

\begin{tabular}{l|l} 
Type I & Clearly circumscribed and dense \\
\hline Type II & Circumscribed, translucent, cloudy, and dense \\
\hline Type III & Translucent and cloudy without definite circumscription \\
\hline
\end{tabular}



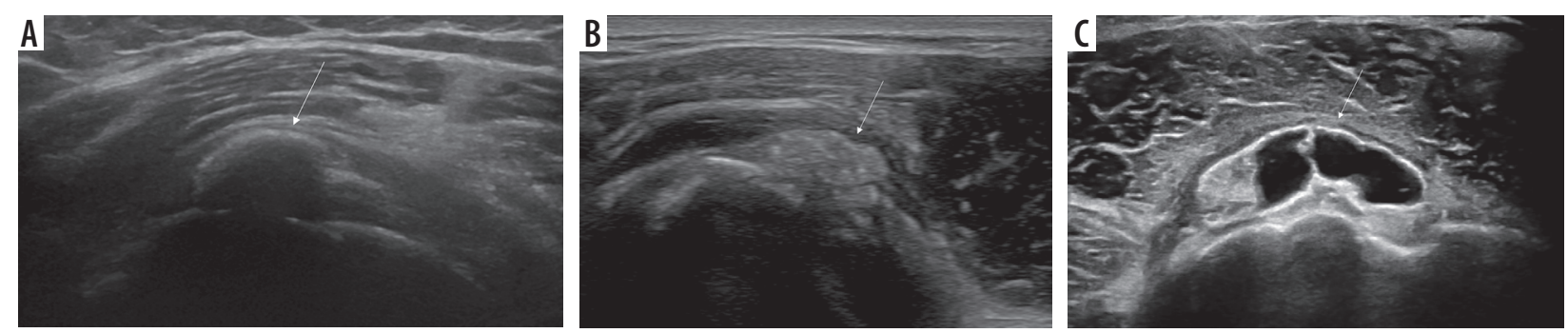

Figure 2. Ultrasound images showing hard (A), soft (B), and fluid (C) calcification (arrows)

- type III calcification: difficult to diagnose for their slightly isoechogenic pattern without posterior acoustic shadow.

Sconfienza et al. [6] classification is based on the degree of hardness usually encountered during a dry needling interventional procedure (Figure 2):

- hard calcifications appear hyperechoic with strong acoustic shadowing;

- soft calcifications appear hyperechoic or slightly isoechoic without acoustic shadowing;

- fluid calcifications appear as hypo/anechoic deposits without acoustic shadowing.

Colour Doppler can be useful in the identification of the phlogistic phase [17] that occurs during the formative and resorptive stage with a direct correlation between Doppler signal and symptoms [18].

There has been innovation in progress regarding the utility of shear-wave sonoelastography, which could be useful for the evaluation of which calcific deposits might be treated on the basis of different hardness values.

\section{Magnetic resonance}

Magnetic resonance (MR) is considered the gold standard imaging modality for the evaluation of the shoulder, particularly in case of RC pathologies [19,20]; although the low number of resonating atoms of calcific crystal usually leads to low accuracy in the detection of CT [21].

On MR, calcifications generally appear as areas of low signal on all sequences within the tendon (Figure 3) [22].

Nörenberg et al. [23] evaluated the diagnostic performance of susceptibility-weighted imaging (SWI) for the detection of CT in comparison to CR. These authors demonstrated $98 \%$ and $96 \%$ of sensitivity and specificity, respectively, in the identification of calcifications when compared with CR.

However, MR plays an important role in the evaluation of surrounding oedema, which is directly proportional to the patient's symptoms, and in the detection of associated tendon tears. High signal intensity of oedematous changing could lead to a false positive findings of tears [6].

\section{Conservative treatments}

It includes manual physical therapy with exercises improving articular ROMs, and the administration of sys- tematic NSAIDs in acute phase for the management of symptoms [24].

Injections of corticosteroids into the SASD bursa may be used to reduce the patient's pain due to sub-acromial impingement and bursal inflammation.

If the pain lasts for more than 6 months, conservative treatment is considered to have failed.

\section{Extracorporeal shock wave therapy}

Extracorporeal shock wave therapy (ESWT) is a minimally invasive option for the treatment of calcific tendinitis [25]. The underlying mechanism of ESWT on RCCT is still debated, but the therapeutic mechanisms of ESWT seem to be caused by:

- mechanical effect due to increasing pressure within the therapeutic focus that causes calcific fragmentation;

- inflammatory effect due to mechanical irritation determining activation of proinflammatory factors with leukocyte recruitment and phagocytosis [26];

- analgetic effect due to the inhibition of pain receptors [27].

Many studies have demonstrated that the therapeutic outcome of ESWT depends on the amount of energy applied, the number of treatments, and the different characteristics of the calcific deposit [28].

There is evidence that ESWT, in association with needling procedure, leads to higher therapeutic outcome compared with ESWT alone [29].

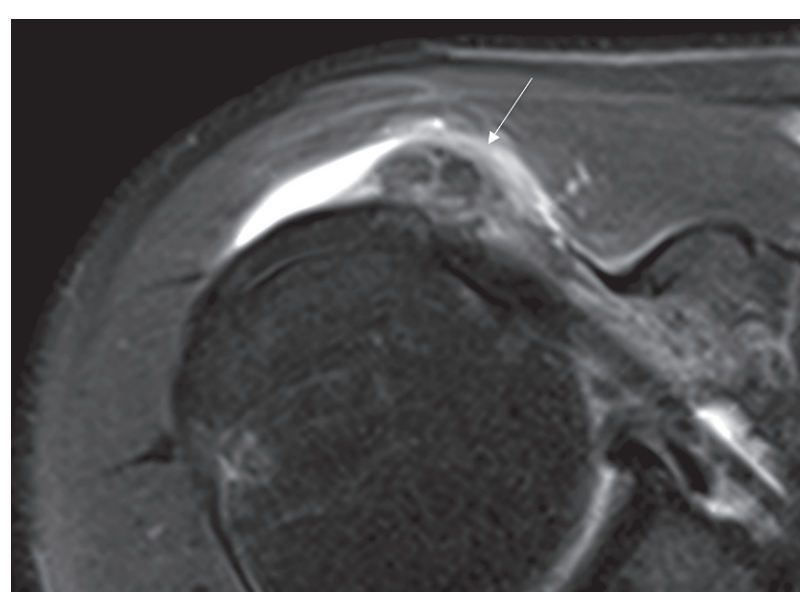

Figure 3. Axial DP fat-sat image showing nuclei of low signal intensity (arrow) within subscapularis tendon. A phlogistic reaction of sub-acromialsub-deltoid bursa is also present 


\section{Ultrasound-guided percutaneous irrigation}

In recent years, US-PICT has become the gold standard approach in cases of RCCT because of its safety, low invasiveness, and efficacy in calcific fading when compared to shock waves, because crystal deposits are drained outside the tendon [30-32]. This interventional technique brings significant pain relief with low incidence of minor complications such as vasovagal reaction and rare septic bursitis [33]. The procedure does not require any hospitalization and is performed under local anaesthesia, and for these reasons the patient can return home about 30 minutes after the procedure; furthermore, there is no need for immobilization, and patients return to their normal life 24 hours after the treatment. Surgical procedures may be associated with a significant psychological burden that potentially correlates with anxiety.

A recent study regarding patient experience during US-PICT reports mild procedural discomfort and overall satisfaction both immediately after treatment and after 3 months [34].

Well-demarcated hyperechoic foci with a weak posterior shadow or calcification with a central semiliquid consistency are the preferable deposit types dissolvable with US-PICT [35].

Not indicated calcification patterns include the following:

- small calcification less than $5 \mathrm{~mm}$;

- fragmented calcification;

- cluster of calcifications inside SASD;

- intra-osseous migration of calcification.

Different approaches have been reported in the literature, which differ from each other due to the number of needles used.

The single-needle technique seems to be more appropriate for the treatment of soft or fluid calcifications [36] while the 2-needle approach might be more appropriate in cases of harder deposits [37]. Regarding the procedure, the patient is positioned semi-supine to prevent vasovagal reaction with the arm extended along the body in a slightly internal or external rotation according to the affected tendon. Subsequently, an antiseptic solution is used to prepare the skin and US probe, and up to $10 \mathrm{ml}$ of local anaesthetic (usually lidocaine) is injected in the SASD bursa, near the calcific deposits and along the needle trajectory. All techniques required 16-gauge $(G)$ needles, while smaller spinal needles of 18-20 G could be used to resolve obstruction inside the larger needle.

In case of the 2-needle technique (Figure 4), the first needle is inserted with an in-plane approach and parallel to the probe into the caudal portion of the calcification with the needle bevel open upwards, while the second needle is inserted into the upper portion of the calcification

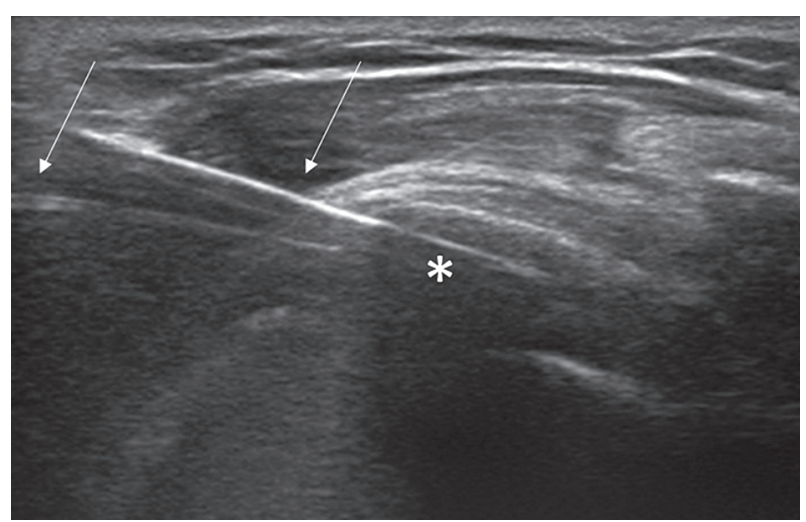

Figure 4. Image of 2-needle ultrasound-guided percutaneous irrigation of calcific tendinopathy (US-PICT) procedure showing needle tips (arrow) into the calcific deposit (asterisk)

parallel and superficial to the first one, with its bevel in the opposite direction in order to create a close circuit [38]. Continual intermittent pressure is made to progressively fill the calcification with saline solution to dissolve its core.

If a single-needle technique is used, the needle tip is inserted with an in-plane approach into the central portion of calcification, and an intermittent pushing procedure is done to wash out calcium material that refluxes inside the syringe [39].

In case of hard calcifications, the use of warm saline solution can reduce treatment duration, facilitate calcium dissolution, and reduce the incidence of post-procedural bursitis [40].

When only the calcification shell remains, a series of tendon perforations are performed under US guidance on the degenerated tendon to fragment the deposit and induce local bleeding, which promote growth factors.

At the end of the procedure an injection of low-solubility steroid into the SASD bursa is performed. The use of colour Doppler may help radiologists with less experience in injecting the drugs inside the correct site [41].

\section{Conclusions}

RCCT is one of the major causes of RC pain and disability, and it can be easily diagnosed with CR and/or US modalities. Even if resolution of calcific deposits occurs spontaneously, a safe and effective procedure for treating RCCT accelerating the reabsorption process. Radiologists must know this mini-invasive US-guided procedure as an alternative to surgical techniques, which are more invasive and prone to postoperative complications.

\section{Conflict of interest}

The authors report no conflict of interest. 


\section{References}

1. Chianca V, Di Pietto F, Zappia M, et al. Musculoskeletal ultrasound in the emergency department. Semin Musculoskelet Radiol 2020; 24: 167-174.

2. Bosworth BM. Calcium deposits in the shoulder and subacromial bursitis: a survey of 12,122 shoulders. J Am Med Assoc 1941; 116 2477-2482.

3. Fabbro E, Ferrero G, Orlandi D, et al. Rotator cuff ultrasound-guided procedures: technical and outcome improvements. Imaging Med 2012; 4: 649-656.

4. Della V, Emilio V, Bassi M. Migration of calcium deposits into subacromial - subdeltoid bursa and into humeral head as a rare complication of calcifying tendinitis: sonography and imaging. J Ultrasound 2015; 18: 259-263.

5. Zhang T, Duan Y, Chen J, Chen X. Efficacy of ultrasound-guided percutaneous lavage for rotator cuff calcific tendinopathy: A systematic review and meta-analysis. Medicine 2019; 98: e15552.

6. Chianca V, Albano D, Messina C, et al. Rotator cuff calcific tendinopathy: from diagnosis to treatment. Acta Biomed 2018; 89: 186-196.

7. McLaughlin HL. Lesions of the musculotendinous cuff of the shoulder: III. Observations on the pathology, course and treatment of calcific deposits. Ann Surg 1946; 124: 354-362.

8. Bishop WA. Calcification of the supraspinatus tendon. Arch Surg 1939; 39: 231

9. Rui YF, Lui PPY, Chan LS, et al. Does erroneous differentiation of tendon-derived stem cells contribute to the pathogenesis of calcifying tendinopathy? Chin Med J 2011; 124: 606-610.

10. Lui PPY. Histopathological changes in tendinopathy-potential roles of BMPs? Rheumatol 2013; 52: 2116-2126.

11. Uhthoff HK, Sarkar K. Calcifying tendinitis. Baillieres Clin Rheumatol 1989; 3: 567-581.

12. Albano D, Coppola A, Gitto S, et al. Imaging of calcific tendinopathy around the shoulder: usual and unusual presentations and common pitfalls. Radiol Med 2021; 126: 608-619.

13. Sansone V, Maiorano E, Galluzzo A, Pascale V. Calcific tendinopathy of the shoulder: clinical perspectives into the mechanisms, pathogenesis, and treatment. Orthop Res Rev 2018; 10: 63-72.

14. Sconfienza LM, Albano D, Allen G, et al. Clinical indications for musculoskeletal ultrasound updated in 2017 by European Society of Musculoskeletal Radiology (ESSR) consensus. Eur Radiol 2018; 28: 5338-5351.

15. Corvino A, Corvino F, Liccardo A, Tafuri D. Calcific tendinopathy of supraspinatus tendon in elite volleyball players: a point-of-care ultrasound diagnosis. Journal of Human Sport and Exercise 2021; 16: 37-52.

16. Bianchi S, Martinoli C. Ultrasound of the musculoskeletal system. Berlin, Heidelberg: Springer Berlin Heidelberg; 2007.

17. Gitto S, Messina C, Chianca V, et al. Superb microvascular imaging (SMI) in the evaluation of musculoskeletal disorders: a systematic review. Radiol Medica 2020; 125: 481-490.

18. Chiou HJ, Chou YH, Wu JJ, et al. Evaluation of calcific tendonitis of the rotator cuff: role of color Doppler ultrasonography. J Ultrasound Med 2002; 21: 289-295.
19. Zappia M, Chianca V, Di Pietto F, et al. Imaging of long head biceps tendon. A multimodality pictorial essay. Acta Biomed 2019; 90: 84-94.

20. Albano D, Chianca V, Zappia M, et al. Imaging of usual and unusual complication of rotator cuff repair. J Comput Assist Tomogr 2019; 43: 359-366.

21. Zubler C, Mengiardi B, Schmid MR, et al. MR arthrography in calcific tendinitis of the shoulder: Diagnostic performance and pitfalls. Eur Radiol 2007; 17: 1603-1610.

22. Chung CB, Gentili A, Chew FS. Calcific tendinosis and periarthritis: classic magnetic resonance imaging appearance and associated findings. J Comput Assist Tomogr 2004; 28: 390-396.

23. Nörenberg D, Ebersberger HU, Walter T, et al. Diagnosis of calcific tendonitis of the rotator cuff by using susceptibility-weighted MR imaging. Radiology 2016; 278: 475-484.

24. Cibek JS, Carcia CR. Presentation and conservative management of acute calcific tendinopathy: a case study and literature review. J Sport Rehabil 2012; 21: 334-342.

25. Mouzopoulos G, Stamatakos M, Mouzopoulos D, Tzurbakis M. Extracorporeal shock wave treatment for shoulder calcific tendonitis: a systematic review. Skeletal Radiol 2007; 36: 803-811.

26. Loew M, Jurgowski W, Mau HC, Thomsen M. Treatment of calcifying tendinitis of rotator cuff by extracorporeal shock waves: a preliminary report. J Shoulder Elb Surg 1995; 4: 101-106.

27. Wang CJ, Wang FS, Yang KD, et al. Shock wave therapy induces neovascularization at the tendon-bone junction. A study in rabbits. J Orthop Res 2003; 21: 984-989.

28. Peters J, Luboldt W, Schwarz W, et al. Extracorporeal shock wave therapy in calcific tendinitis of the shoulder. Skeletal Radiol 2004; 33: 712-718.

29. Orlandi D, Corazza A, Arcidiacono A, et al. Ultrasound-guided procedures to treat sport-related muscle injuries. Br J Radiol 2016; 89: 20150484.

30. Lanza E, Banfi G, Serafini G, et al. Ultrasound-guided percutaneous irrigation in rotator cuff calcific tendinopathy: what is the evidence? A systematic review with proposals for future reporting. Eur Radiol 2015; 25: 2176-2183.

31. Sconfienza LM, Adriaensen M, Albano D, et al. Clinical indications for image-guided interventional procedures in the musculoskeletal system: a Delphi-based consensus paper from the European Society of Musculoskeletal Radiology (ESSR) - Part II, elbow and wrist. Eur Radiol 2020; 30: 2220-2230.

32. Sconfienza LM, Adriaensen M, Albano D, et al. Clinical indications for image-guided interventional procedures in the musculoskeletal system: a Delphi-based consensus paper from the European Society of Musculoskeletal Radiology (ESSR) - part I, shoulder. Eur Radiol 2020; 30: 903-913.

33. Sconfienza LM, Randelli F, Sdao S, et al. Septic bursitis after ultrasound-guided percutaneous treatment of rotator cuff calcific tendinopathy. PM R 2014; 6: 746-748.

34. Albano D, Gambino A, Messina C, et al. Ultrasound-guided percutaneous irrigation of rotator cuff calcific tendinopathy (US-PICT): patient experience. Biomed Res Int 2020; 2020: 3086395. 
35. Messina C, Sconfienza LM. Ultrasound-guided percutaneous irrigation of calcific tendinopathy. Semin Musculoskelet Radiol 2016; 20: 409-413.

36. Orlandi D, Fabbro E, Mauri G, et al. RE: A simple technique to restore needle patency during percutaneous lavage and aspiration of calcific rotator cuff tendinopathy. PM R 2013; 5: 633.

37. Farin PU, Jaroma H, Soimakallio S. Rotator cuff calcifications: treatment with US-guided technique. Radiology 1995; 195: 841-843.

38. Sconfienza LM, Serafini G, Sardanelli F. Treatment of calcific tendinitis of the rotator cuff by ultrasound-guided single-needle lavage technique. Am J Roentgenol 2011; 197: W366-W366.
39. Sconfienza LM, Viganò S, Martini C, et al. Double-needle ultrasound-guided percutaneous treatment of rotator cuff calcific tendinitis: tips \& tricks. Skeletal Radiol 2013; 42: 19-24.

40. Sconfienza LM, Bandirali M, Serafini G, et al. Rotator cuff calcific tendinitis: does warm saline solution improve the short-term outcome of double-needle US-guided treatment? Radiology 2012; 262: 560-566.

41. Albano D, Chianca V, Tormenta S, et al. Old and new evidence concerning the crucial role of ultrasound in guiding intra-articular injections. Skeletal Radiol 2017; 46: 963-964. 